\title{
Subject strategies and the effect of MA on learning rate and acquisition patterns of serial verbal material ${ }^{12}$
}

WILLIAM R. BLOUNT AND LAIRD W. HEAL

GEORGE PEABODY COLLEGE FOR TEACHERS

Thirty retardates from three MA levels were given a test of $10 \mathrm{CVC}$ trigrams in a serial anticipation task. MA interacted with serial position; low MA having more difficulty with the positions right of center. It appeared that the discrepancy between the Ellis et al (1959), study and that by Woodruff $\mathcal{E}$ Pielstick (1965) could be accounted for in terms of the dependent variable (errors vs. trials to acquisition) used in the two studies and the fact that Woodruff E Pielstick confounded position with trigrams.

There is some disagreement regarding the relationship between acquisition pattern (serial position curve) of serial verbal material and ability level. Ellis et al (1959), using retarded, normal, and superior Ss of the same CA in a serial verbal learning task (Experiment 1) with 10 lists of 10 nouns as stimuli, report greater "bowing" (more difficulty with positions six, seven, and eight) of the bell-shaped curve as IQ decreased. Woodruff \& Pielstick (1965), using CVC trigrams with normal and gifted children from the third, fifth, and seventh grades, reported for their low association list that only the fifth and seventh grade gifted children produced the bell-shaped curve, while the remaining groups showed a "leveling" effect in that there were no differences between the difficulty of those trigrams in the middle of the list and those at the end. A similar but non-significant effect was noted for the high association list. They suggested that acquisition patterns varied with ability level. If low ability Ss tend to "level" and high ability Ss tend to show the bell-shaped curve, the present study aimed at investigating whether or not even lower ability Ss (retardates) would give a linear function as an acquisition pattern. A second purpose was to try to account for the differences between the Ellis et al study and the Woodruff and Pielstick study. However, in this replication of the Woodruff and Pielstick study, several changes were made. First, five lists of the trigrams were used to unconfound serial position and trigrams (trigrams and positions were confounded in the Woodruff and Pielstick study); second, lower ability groups were used; third, only the high association value list was used; and fourth, dependent variables of errors, trials to criterion, and trials to acquisition (the first trial after the last error for a given trigram) were used to note any effect the dependent variable might have. Subjects

Three MA groups (5, 7, and 9) of 10 retarded Ss each were randomly selected from the available subject pool at Clover Bottom Hospital and School. Mean MAs were
5 yrs. 7 mos. (MA 5), 6 yrs. 10 mos. (MA 7), and 9 yrs. 2 mos. (MA 9) with standard deviations (SDs) of 6 mos., 2 mos., and 7 mos. Mean IQs (Binet) were 37.2, $(\mathrm{SD}=5.4), 49.1(\mathrm{SD}=8.4)$, and $58.0(\mathrm{SD}=7.3)$ respec ${ }^{1}$ ively. Mean CAs were 19 yrs. 5 mos. (MA 5), 19 yrs. 9 mos. (MA 7), and 22 yrs. 5 mos. (MA 9) with SDs of 5 yrs., 4 yrs. 10 mos., and 5 yrs. 7 mos. Mean lengths of institutionalization for the groups were $7 \mathrm{yrs} .10$ mos. (MA 5), 4 yrs. 11 mos. (MA 7) and 9 yrs. 7 mos. (MA 9) with SDs of 4 yrs. 1 mo., 2 yrs. 9 mos., and 5 yrs., respectively.

\section{Procedure}

Ss were run individually. Each learned one of five lists of the $10 \mathrm{CVC}$ trigrams (MON, KAD, SIC, PUF, WIS, GEL, BAK, JIF, CYN, NAH) from the Woodruff and Pielstick high association list (85\% according to the Archer, 1960, norms). Ss were required to spell the trigrams (typed in capital letters) in an anticipation procedure. The five lists were constructed by randomly assigning the trigrams to numbers from 1 to 10 and then ordering the trigrams into lists according to five randomly chosen rows of a 10 by 10 balanced Latin square, constructed so that each word followed every other word once and occurred in a given position once. Six Ss, two from each MA group, were randomly assigned to each list. A Lafayette memory drum (model No. 303) was used with a $2 \mathrm{sec} . / 2 \mathrm{sec}$. presentation rate with an $8 \mathrm{sec}$. intertrial interval. Criterion was one perfect recitation of the list.

\section{Results and Discussion}

The two major dependent variables were errors (Fig. 1) and trials to acquisition (Fig. 2). MA by List by Position was analyzed using both dependent va riables. The results of these analyses ( $p<.01$ for all effects) were as follows. Using trials to acquisition as the dependent variable, only Position was significant, $F=$ 21.97, $\mathrm{df}=9 / 135$; for the same analysis using errors as the dependent variable, both Position and MA by Position were significant $(F=59.43, \mathrm{df}=9 / 135$ and $\mathrm{F}=$ $2.09, \mathrm{df}=18 / 135$, respectively). Inspection of Fig. 1 shows that this interaction is attributable to the relatively large number of errors for low MASs in the middle and late middle positions of the list. One interpretation of this interaction is that lower ability Ss are more susceptible to the associative interference that is usually assumed to underlie the serial position bow. In addition to the analyses of MA by List by Position, the data were also analyzed in terms of MA by List by Trigram. For this breakdown, using trials to acquisition, 


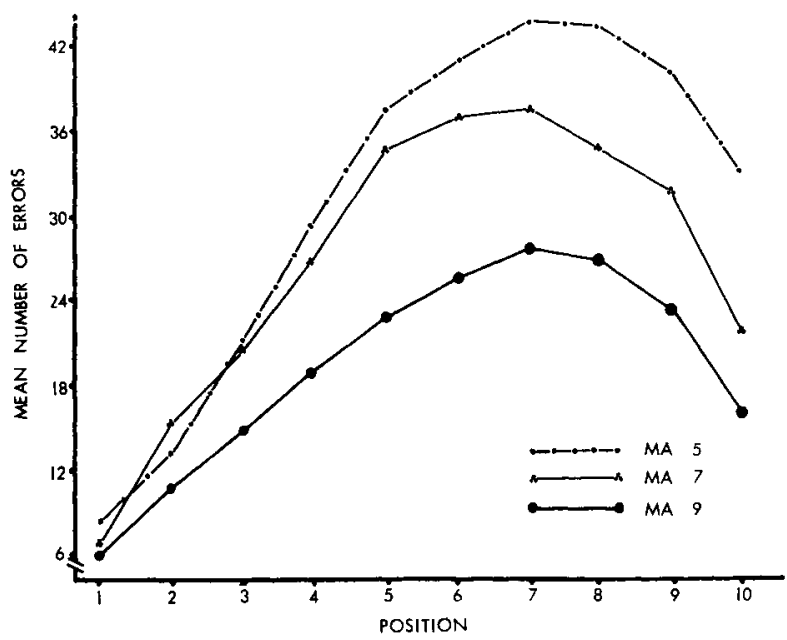

Fig. 1. Semantic representation of the MA by Position interaction found using number of errors as the de pendent variable.

the effects of Trigram $(F=4.41, d f=9 / 135)$ and List by Trigram $(F=5.52, \mathrm{df}=36 / 135)$ were significant; for the same analysis using errors as the dependent variable, Trigram $(F=4.41, d f=9 / 135)$ and List by Trigram $(F=$ 14.34, $\mathrm{df}=36 / 135$ ) were again significant. The MA by List analysis, using trials to criterion as the dependent variable, gave no significant effects. No other effects were significant at the .05 level in any analysis.

IQ did not correlate with either errors or trials to criterion, but MA did ( -.41 and -.38 respectively). Thus, the ability level differences found in the present study are attributed to MA and not to IQ. It seems probable that with greater ranges both MA and IQ will produce these differences.

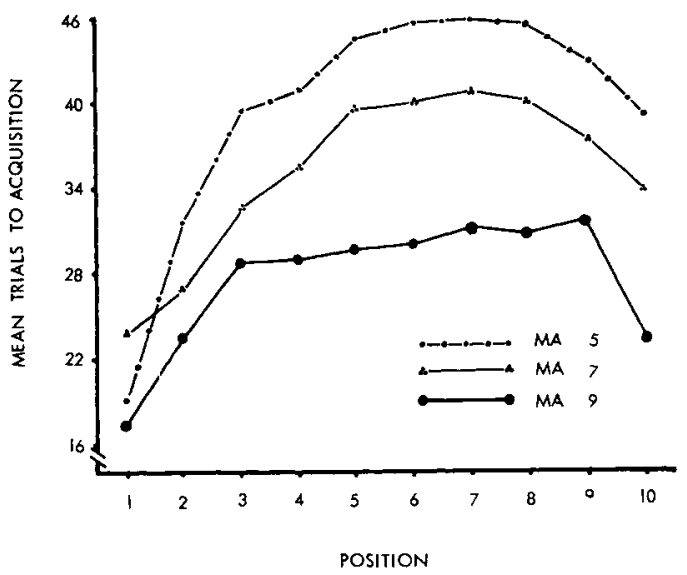

Fig. 2. Semantic representation of the MA by Position interaction found using trials to acquisition as the dependent variable.
The acquisition patterns (serial position curves with trials to acquisition as the dependent variable) were noted for all Ss. Sixteen Ss showed a "bowed" serial position. Eight Ss showed a "sudden" acquisition pattern, making at least four errors on their last precriterion trial. Four of these eight Ss missed at least seven trigrams on the trial directly preceding criterion. Three Ss demonstrated a "linear"' acquisition pattern, reaching criterion on their first seven positions before their last three. Three Ss could not be classified. The effect of these last two patterns was to flatten the serial position curves, especially in the late positions, when trials to acquisition was the dependent variable. These acquisition patterns were not common to any particular MA group or list.

There is lack of support for the original hypothesis that the low MA Ss of the present study would produce a "linear" acquisition pattern. Only four Ss showed such a pattern.

The present data suggest a resolution of the discrepant serial position functions of Ellis et al (bowed curve) and of Woodruff and Pielstick (leveled curve for low ability, bowed for high). First, it appears that Woodruff and Pielstick's dependent variable (trials to acquisition) tended to reduce serial position differences, especially in the later positions, because of the Ss' strategies that were reflected in this measure. Eleven of the present $30 \mathrm{Ss}$ had acquisition patterns ("sudden" or "linear") that served to raise trial scores for the words at the end of the list and lower these scores in the middle. The errors measure, used by Ellis et al, is relatively unaffected by these $\mathrm{Ss}^{\prime}$ strategies (cf. Figs. 1 and 2). Second, it seems that the confounding of trigrams with position was partially responsible for the leveling that Woodruff and Pielstick reported. Our analyses indicated that the trigrams differed significantly in difficulty (cf. main effects of trigram). In fact, the last two trigrams on their list (CYN, NAH) were the hardest for our Ss.

\section{References}

Archer, E. J. A re-evaluation of the meaningfulness of all possible CVC trigrams. Psychol. Monogr., 1960, 74, No. 10 (Whole No. 497).

Ellis, N. R., Pryer, Margaret W., Distefano, M. K., \& Pryer, R. L. Learning in mentally defective, normal, and superior children. Amer. J. ment. Defic., 1959, 64, 725-734.

Woodruff, A. B., \& Pielstick, N. L. Effect of age and IQ on rate of learning and pattern of acquisition of lists of trigrams. Psychon. Sci., 1965, 3, 145-146.

\section{Notes}

1. Grateful acknowledgement is given the administration and staff of Clover Bottom Hospital and School, Nashville, Tennessee, for their cooperation.

2. This study was supported in part by NICHD grant HD-43 and NIMH grant 12701-01. 